\title{
Isospin mixing and Fermi transitions: Self-consistent deformed mean field calculations and beyond
}

\author{
R. Álvarez-Rodríguez, E. Moya de Guerra, and P. Sarriguren \\ Instituto de Estructura de la Materia, Consejo Superior de Investigaciones Científicas, Serrano 123, E-28006 Madrid, Spain
}

(Received 29 November 2004; published 20 April 2005)

\begin{abstract}
We study Fermi transitions and isospin mixing in an isotopic chain $\left({ }^{70-78} \mathrm{Kr}\right)$ considering various approximations that use the same Skyrme-Hartree-Fock single-particle basis. We study Coulomb effects as well as the effect of BCS and quasiparticle random phase approximation (QRPA) correlations. A measure of isospin mixing in the approximate ground state is defined by means of the expectation value of the isospin operator squared in $N=Z$ nuclei (which is generalized to $N \neq Z$ nuclei). Starting from a strict Hartree-Fock approach without Coulomb interaction, it is shown that the isospin breaking is negligible, on the order of a few per thousand for $(N-Z)=6$, increasing to a few percent with Coulomb interaction. Pairing correlations induce rather large isospin mixing and Fermi transitions of the forbidden type ( $\beta^{-}$for $N \leqslant Z$, and $\beta^{+}$for $N \geqslant Z$ ). The enhancement produced by BCS correlations is compensated to a large extent by QRPA correlations induced by isospin-conserving residual interactions that tend to restore isospin symmetry.
\end{abstract}

DOI: 10.1103/PhysRevC.71.044308 PACS number(s): 21.60.Jz, 23.40.Hc, 21.10.Hw, 27.50.+e

\section{INTRODUCTION}

It is well known [1] that the self-consistent mean field Hamiltonian breaks the symmetries of the exact Hamiltonian. The best-known example is the breaking of rotational invariance by the self-consistent mean field of deformed nuclei. Rotational invariance breaking often leads to ground states of the Hartree-Fock (HF) or Hartree-Fock-Bogoliubov (HFB) type with large expectation values of the squared angular momentum operator. For heavy well-deformed even-even nuclei one typically has $[2,3]$

$$
\left\langle J_{\perp}^{2}\right\rangle \geq 100
$$

where $J_{\perp}$ is the angular momentum operator component perpendicular to the symmetry axis and \langle\rangle means expectation value in the HF or HFB ground state. In these instances the $\mathrm{HF}$ or HFB wave function can be interpreted as a wave packet from which the ground-state rotational band can be obtained by angular momentum projection. The angular momentum projection can be carried out through an expansion in powers of $1 /\left\langle J_{\perp}^{2}\right\rangle,[4]$ which, to lowest order, provides a factorization approximation formally identical to that of Bohr and Mottelson [5].

Unlike rotational invariance in ordinary space, rotational invariance in isospin space is not an exact symmetry of the actual total nuclear Hamiltonian. The Coulomb force is nonisoscalar, and thus the actual nuclear states may have isospin mixing. Isospin mixing in the ground state may allow for Fermi (F) transitions in $N=Z$ nuclei, and it is a point of present debate because of its implications on parity violation experiments on ${ }^{12} \mathrm{C}$ [6], as well as in the analysis of superallowed Fermi transitions as a test of the unitarity of the Cabbibo-Kobayashi-Maskawa matrix [7].

Nonetheless, even with isoscalar nuclear Hamiltonians, the self-consistent mean field may break isospin invariance, particularly the self-consistent quasiparticle mean field (HF+BCS or HFB). Therefore in studying Fermi transitions and isospin mixing in the nuclear ground state, it is important to know to what extent the theoretical results respond to realistic properties of the interactions used in the calculations, or rather to spurious mean field contributions.

It is important in many respects to know the value of the quantity

$$
\left\langle T_{\perp}^{2}\right\rangle=\left\langle T^{2}\right\rangle-\left(\frac{N-Z}{2}\right)^{2}
$$

If $\left\langle T_{\perp}^{2}\right\rangle$ is large when $N=Z$, one may consider, in analogy to the case $\left\langle J_{\perp}^{2}\right\rangle \gg 1$, that the mean field ground state is a superposition of several $T$ eigenstates. In such a case, one may generate a corresponding isospin rotational band by isospin projection [8], in analogy to the aforementioned angular momentum projection method, or one may even use an isospin-cranked mean field approximation. The latter method has been discussed by Wyss and coworkers [9] in a somewhat different context. In contrast, if $\left\langle T_{\perp}^{2}\right\rangle$ is small $\left(\left\langle T_{\perp}^{2}\right\rangle \lesssim 1\right.$ for $N=Z$ ), it means that isospin mixing is small. In this case the mean field ground state is nearly an eigenstate of total isospin, no isospin projection may be required, and the influence of isospin mixing forces can be reliably studied.

In the shell model context [10] large isospin mixing forces (or matrix elements) have been considered. In this paper we restrict our consideration to the Coulomb force as treated in standard mean field calculations [11].

We study Fermi transitions and isospin properties of ground states of several nuclei, from stable to proton-rich isotopes, at various levels of approximation. We first consider mean field ground states with and without pairing correlations and with and without isospin breaking interactions (Coulomb force). Next we take into account isospin-dependent residual interactions and consider quasiparticle random phase approximation(QRPA) correlated ground states. The isospin restoring effect of QRPA correlations due to isospin-conserving residual interactions is discussed.

Other HF, Tamm-Dancoff approximation (TDA), or RPA studies of isospin impurities in the ground states of several $N=$ $Z$ nuclei, as well as studies of the effect of such impurities on superallowed Fermi and Gamow-Teller $\beta$ decay, can be found 

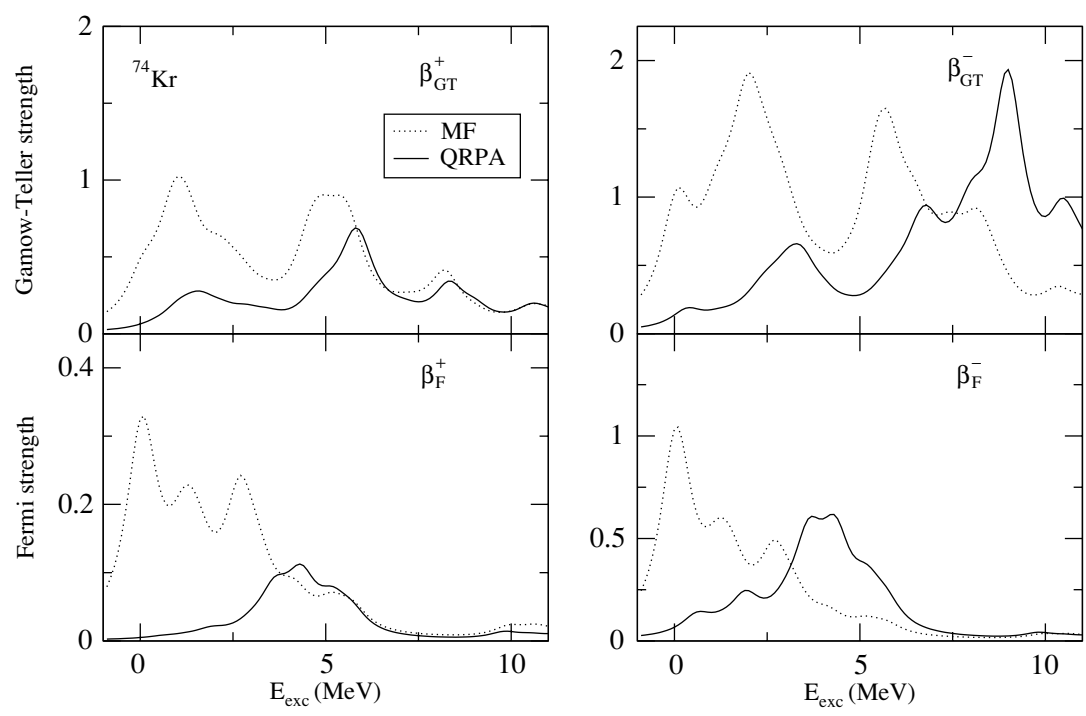

FIG. 1. Comparison of $\beta^{ \pm}$Gamow-Teller and Fermi strength distributions in both the mean field (MF) approximation and the QRPA in the case of ${ }^{74} \mathrm{Kr}$.

in Refs. [12,13] and references therein. The latter works show that a simple perturbative treatment of the Coulomb interaction using the same unperturbed wave functions for neutrons and protons will lead to an overestimate of isospin mixing and that the use of self-consistent solutions is essential in calculating the values of isospin mixing probabilities. The consistency between the interaction producing the single-particle spectrum and the strength of the residual particle-hole interaction is an important ingredient in our calculations. This consistency has been shown to be essential in many RPA calculations of giant resonances $[12,14,15]$, as well as in double $\beta$ decay [16].

The paper is organized as follows: In Sec. II we present results on Fermi strength distributions. In Sec. III we compare $\left\langle T_{\perp}^{2}\right\rangle$ values to $\left\langle J_{\perp}^{2}\right\rangle$ values in the mean field approach and we discuss $\left\langle T_{\perp}^{2}\right\rangle$ values in different approaches. In Sec. IV we summarize the main conclusions.

\section{FERMI STRENGTH DISTRIBUTIONS}

In previous publications we studied $\beta$-decay strength functions $[17,18]$ of several isotopic chains in the $A \simeq$ 60-80 region, within the mean field context and beyond. We performed QRPA calculations on top of a quasiparticle basis obtained from a self-consistent deformed Hartree-Fock approach with density-dependent Skyrme forces. In this work we use the Skyrme force SG2 [19] as a representative of these forces. We use Gamow-Teller (GT) and Fermi residual interactions consistent with the mean field single-particle basis, both being derived from the same two-body Skyrme interactions. In those works attention was focused on GT strength distributions that are dominant (see Fig. 1). At variance with standard shell-model calculations [20], in our self-consistent mean field-based calculations [17,18], isospin is not an exact quantum number, and one may wonder how large the effect of isospin breaking can be. We investigate this question here, focusing on Fermi transitions where isospinbreaking effects can be expected to be of most importance.
The Fermi strength distribution can be written as

$$
B^{F^{ \pm}}(E)=\sum_{f} \delta\left(E-E_{f}\right) B_{f}^{F^{ \pm}}
$$

with

$$
B_{f}^{F^{ \pm}}=\left|F_{f}^{ \pm}\right|^{2}, \quad F_{f}^{ \pm}=\left\langle f\left|T_{ \pm}\right| 0\right\rangle,
$$

where $T_{ \pm}$are the rising and lowering isospin operators, $|0\rangle$ represents the ground state, and $|f\rangle$ are the proton-neutron (pn) excited states.

For the even-even system both initial and final states are represented by factorized wave functions of the BohrMottelson type with a common collective wave function $D_{00}^{0}$ and intrinsic wave functions $|0\rangle$ and $|f\rangle$. In the deformed mean field approximation $|f\rangle$ represents a one-particle, one-hole or a two-quasiparticle excitation, connected to the HF or HFB ground state of the parent nucleus by the $T_{ \pm}$operator,

$$
\begin{aligned}
& F_{f}^{+}=2 \sum_{i p i^{\prime} n}\left\langle i^{\prime} \mid i\right\rangle u_{i^{\prime} n} v_{i p}\left\langle f\left|\alpha_{i^{\prime} n}^{+} \alpha_{\bar{l} p}^{+}\right| 0\right\rangle, \\
& F_{f}^{-}=2 \sum_{i p i^{\prime} n}\left\langle i^{\prime} \mid i\right\rangle^{*} v_{i^{\prime} n} u_{i p}\left\langle f\left|\alpha_{i p}^{+} \alpha_{\bar{l}^{\prime} n}^{+}\right| 0\right\rangle .
\end{aligned}
$$

The single-particle states $|i\rangle$ are characterized by the eigenvalues $\Omega_{i}$ of $J_{z}$ and by parity $\pi_{i}$. They are expanded in terms of the eigenstates of an axially symmetric harmonic oscillator in cylindrical coordinates, given in terms of Hermite and Laguerre polynomials $[11,17]$ :

$$
|i\rangle=\sum_{N} \frac{(-1)^{N}+\pi_{i}}{2} \sum_{n_{r}, n_{z}, \Lambda \geqslant 0, \Sigma} C_{N n_{r} n_{z} \Lambda \Sigma}^{i}\left|N n_{r} n_{z} \Lambda \Sigma\right\rangle
$$

with $\Omega_{i}=\Lambda+\Sigma \geqslant 1 / 2$. For each $N$, the sum over $n_{r}, n_{z}, \Lambda$ is extended to the quantum numbers satisfying $2 n_{r}+n_{z}+\Lambda=$ $N$. The sum over $N$ goes from $N=0$ to $N=10$. The overlaps $\left\langle i^{\prime} \mid i\right\rangle$ are given by

$$
\left\langle i^{\prime} \mid i\right\rangle=\sum_{N n_{z} \Lambda \Sigma} C_{N n_{r} n_{z} \Lambda \Sigma}^{i^{\prime}} C_{N n_{r} n_{z} \Lambda \Sigma}^{i} .
$$



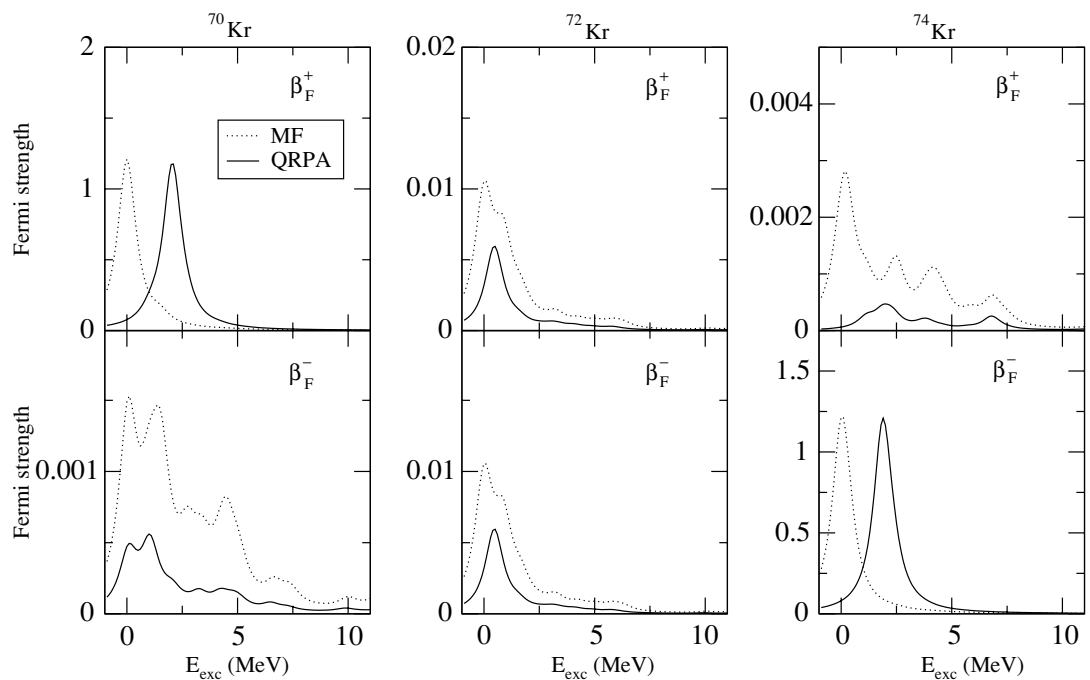

FIG. 2. Fermi strength distributions $\beta^{ \pm}$in ${ }^{70,72,74} \mathrm{Kr}$ plotted as a function of the excitation energy of the daughter nucleus. We compare results obtained from Skyrme Hartree-Fock calculations (dotted lines) and QRPA (solid lines). The results correspond to the case of no Coulomb interaction and pairing gaps approaching zero $(\Delta=0.1 \mathrm{MeV})$.

In Eqs. (2.3) and (2.4) ip $i^{\prime} n$ indicates a proton, neutron state with quantum numbers $i, i^{\prime}$ with $\Omega_{i}=\Omega_{i^{\prime}}>0$ and $v_{i \tau}\left(u_{i \tau}\right)$ the probability amplitude that the single-particle state $i \tau$ be occupied (empty) in the ground state. For each two-quasiparticle excitation $i^{\prime} n, \bar{\imath} p$ the value of the energy is $E_{i^{\prime} n}+E_{\bar{l} p}=E_{f}$. In the limit of no pairing correlations in the ground state (i.e., in the HF limit) $v_{i \tau}$ goes to 1 and 0 for $i \tau$ levels below and above the $\lambda_{\tau}$ Fermi level, respectively. In this limit $E_{f}=\varepsilon_{i^{\prime} n}-\varepsilon_{i p}$ for $\beta^{+}$and $N<$ $Z ; E_{f}=\varepsilon_{i p}-\varepsilon_{i^{\prime} n}$ for $\beta^{-}, N>Z$. We use the convention $\tau=p=-1 / 2, \tau=n=1 / 2$ (i.e., $t_{+}|p\rangle=|n\rangle, t_{-}|n\rangle=p$ ).

In the QRPA method $|f\rangle$ represents a $p n$-QRPA phonon state and $|0\rangle$ the QRPA correlated ground state. The expression for the Fermi strength is then given by

$$
F_{f}^{+}=2 \sum_{i p i^{\prime} n}\left\langle i^{\prime} \mid i\right\rangle\left[u_{i^{\prime} n} v_{i p} X_{i^{\prime} n i p}^{f}+v_{i^{\prime} n} u_{i p} Y_{i^{\prime} n i p}^{f}\right],
$$

with $X_{i^{\prime} n \text { ip }}^{f}$ and $Y_{i^{\prime} n i p}^{f}$ the forward and backward amplitudes for the two-quasiparticle component $i^{\prime} n$ ip of the QRPA mode $|f\rangle=\Gamma_{f}^{+}|0\rangle$, of energy $E_{f}=\omega_{f}$.

An analogous expression is obtained for $F_{f}^{-}$by changing $X$ amplitudes with $Y$ amplitudes. The calculations of these amplitudes have been done as described in Ref. [17]. We use separable residual interactions as those derived in Ref. [17]. We note that the residual Fermi interaction $\left(\vec{\tau}_{1} \cdot \vec{\tau}_{2}\right)$ [as well as the $\left.\operatorname{GT}\left(\vec{\sigma}_{1} \cdot \vec{\sigma}_{2}\right)\left(\vec{\tau}_{1} \cdot \vec{\tau}_{2}\right)\right]$ is an isospin-restoring force and largely eliminates the spurious contributions due to isospin breaking that may be present in mean field calculations.

In Fig. 1 we compare Fermi strengths to GT strengths taking as an example ${ }^{74} \mathrm{Kr}$. The Fermi strength given by Eq. (2.1) and the corresponding expression for the GT strength have been transformed into continuous curves by a folding procedure using Gaussians of $\Gamma=1 \mathrm{MeV}$ width. The strength distributions are plotted versus the excitation energy of the daughter nucleus. The dominance of GT strengths over Fermi strengths is apparent for both $\beta^{+}$and $\beta^{-}$. One clearly sees that the already small mean field Fermi strengths get further reduced in RPA. The general reduction of the strengths in QRPA can be traced back to the isospin-restoring role of the residual interactions, being maximal for the $\beta^{+}$Fermi strength that is strongly reduced. The results shown in this figure are obtained taking into account the Coulomb interaction in the mean field.

In the next figures (Figs. 2-5) we show the role of the Coulomb interaction, as well as that of pairing and RPA correlation effects, on Fermi strengths calculated for the $\mathrm{Kr}$ isotopic chain for $A=70-74$.

In Fig. 2 we show the Fermi strengths obtained in the limit of small pairing $(\Delta=0.1 \mathrm{MeV})$ and no Coulomb force. Starting with ${ }^{70} \mathrm{Kr}$ on the left, we observe that the $\beta^{+}$strength (plotted in the upper panels) rapidly decreases with increasing $\mathrm{A}$, whereas the $\beta^{-}$strength (plotted in the bottom panels) rapidly increases with $A$. We also observe that the strength reduction in going from mean field to QRPA is much stronger in the isospinforbidden cases.

To clarify what we mean by isospin-forbidden cases let us recall that if the ground state $|0\rangle$ is the exact ground state of the system, in the absence of the Coulomb force, it is an eigenstate of the isospin operator with eigenvalues $T_{z}=(N-Z) / 2$ and $T=\left|T_{z}\right|$.

Hence, it is clear that in this case

$$
\begin{aligned}
& T_{+}|0\rangle=\left\{\begin{array}{l}
T_{+}\left|T T_{z}\right\rangle=\sqrt{(Z-N)}\left|T T_{z}+1\right\rangle \text { for } N<Z, \\
0 \quad \text { for } N \geqslant Z,
\end{array}\right. \\
& T_{-}|0\rangle= \begin{cases}T_{-}\left|T T_{z}\right\rangle=\sqrt{(N-Z)}\left|T T_{z}-1\right\rangle \text { for } N>Z, \\
0 & \text { for } N \leqslant Z .\end{cases}
\end{aligned}
$$

Consequently, in Eqs. (2.1) and (2.2) there is a single $|f\rangle$ state, $|f\rangle=\left|T T_{z} \pm 1\right\rangle$ (with $E_{f}=E_{0}$ ), that is accessible by the $\beta^{+}$ (if $N<Z$ ) or the $\beta^{-}$(if $N>Z$ ) operators. Accordingly, we call these isospin-forbidden transitions to the $\beta^{+}$(respectively, $\beta^{-}$) transitions in $\mathrm{Kr}$ isotopes with $A \geqslant 72$ (respectively, $A \leqslant 72$ ). The fact that all isospin-forbidden transitions in Fig. 2 are weak indicates that isospin breaking is weak in the mean field approximation and gets further reduced in QRPA.

As we include the Coulomb interaction, $T$ is no longer an exact quantum number, and isospin breaking (although very weak) becomes an actual property of system. In the 

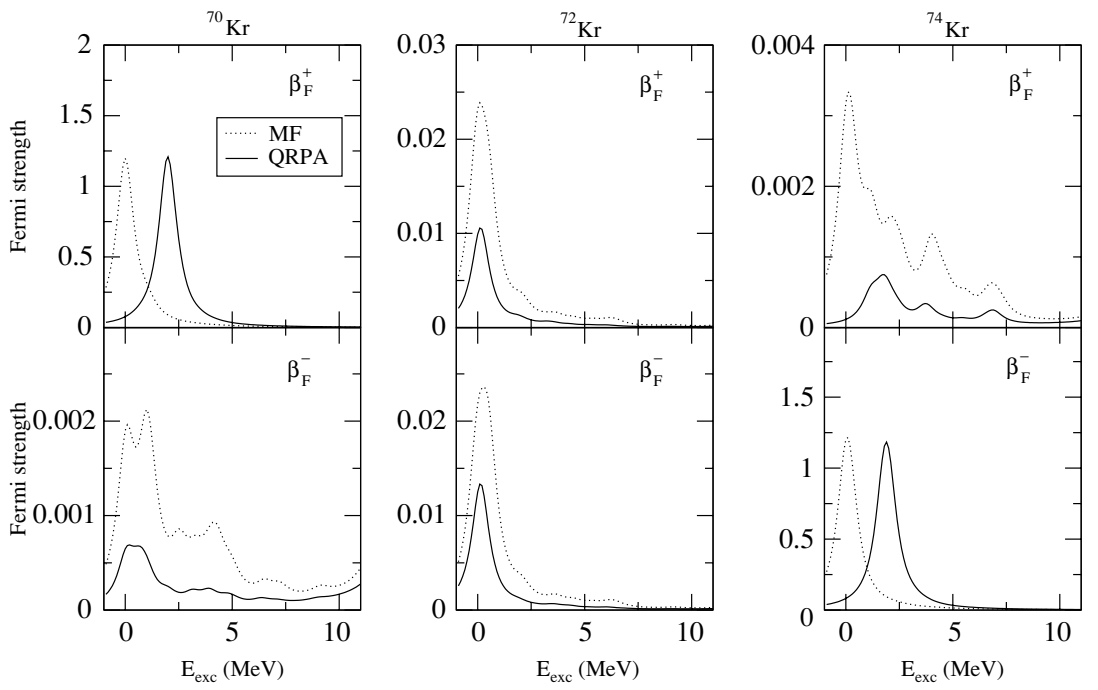

FIG. 3. Same as in Fig. 2 but with Coulomb interaction.

presence of Coulomb interaction Eqs. (2.8) and (2.9) are no longer strictly valid, allowing for $\beta^{ \pm}$transitions in the isospinforbidden cases. When we compare the results in Fig. 3, which we obtained by including the Coulomb force, with the corresponding ones in Fig. 2 we observe an increase of the $\beta^{+}$ ${ }^{72} \mathrm{Kr},{ }^{74} \mathrm{Kr}$ strengths, as well as of the $\beta^{-{ }^{70}} \mathrm{Kr},{ }^{72} \mathrm{Kr}$ strengths. This small increase of the isospin-forbidden transitions of Fig. 2 is solely due to the effect of the Coulomb force on Fermi transition and it is a signature of the corresponding small isospin breaking.

One may also observe that, in the absence of the Coulomb force (Fig. 2), the $\beta^{+}$and $\beta^{-}$strength distributions are identical in ${ }^{72} \mathrm{Kr}$, but the two distributions become somewhat different when the Coulomb force is taken into account (Fig. 3).

Finally, in Fig. 4 we show the $\beta^{+}$and $\beta^{-}$strengths for the same nuclei obtained with realistic pairing gaps $(\Delta \sim$ 1.5 MeV; see Table II). For the allowed transitions $\left(\beta^{+}\right.$in ${ }^{70} \mathrm{Kr}, \beta^{-}$in ${ }^{74} \mathrm{Kr}$ ) one can see by comparison with Fig. 2 that the main effect of pairing is to open up more transition channels and to moderately reduce the strengths of the dominant peaks. More dramatic is the effect of pairing on the isospinforbidden transitions, which grow by orders of magnitude in all cases, even though they remain considerably weaker than the allowed ones. This growth of the isospin-forbidden strengths is mainly connected to the stronger isospin breaking of the quasiparticle mean field with increasing pairing gaps. It is present irrespective of whether or not Coulomb interaction is included in the calculation (see also Fig. 5). One also sees that the QRPA results strongly reduce the strengths of the isospin-forbidden $\beta^{ \pm}$transitions as compared to their mean field values.

\section{ISOSPIN MIXING AND EXPECTATION VALUES OF ISOSPIN OPERATORS}

As mentioned in the Introduction the amount of angular momentum mixing in the mean field ground state of axially symmetric deformed nuclei is measured by the expectation value of the squared angular momentum operator
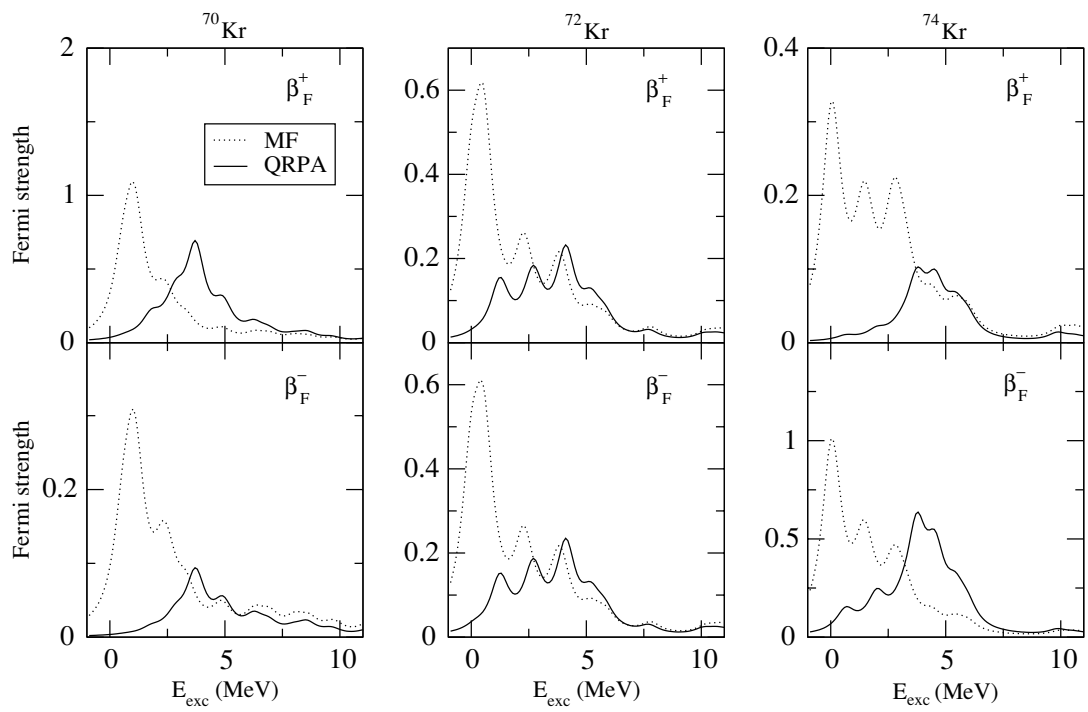

FIG. 4. Same as in Fig. 2 without Coulomb interaction but including pairing correlations with realistic gaps (see Table II). 

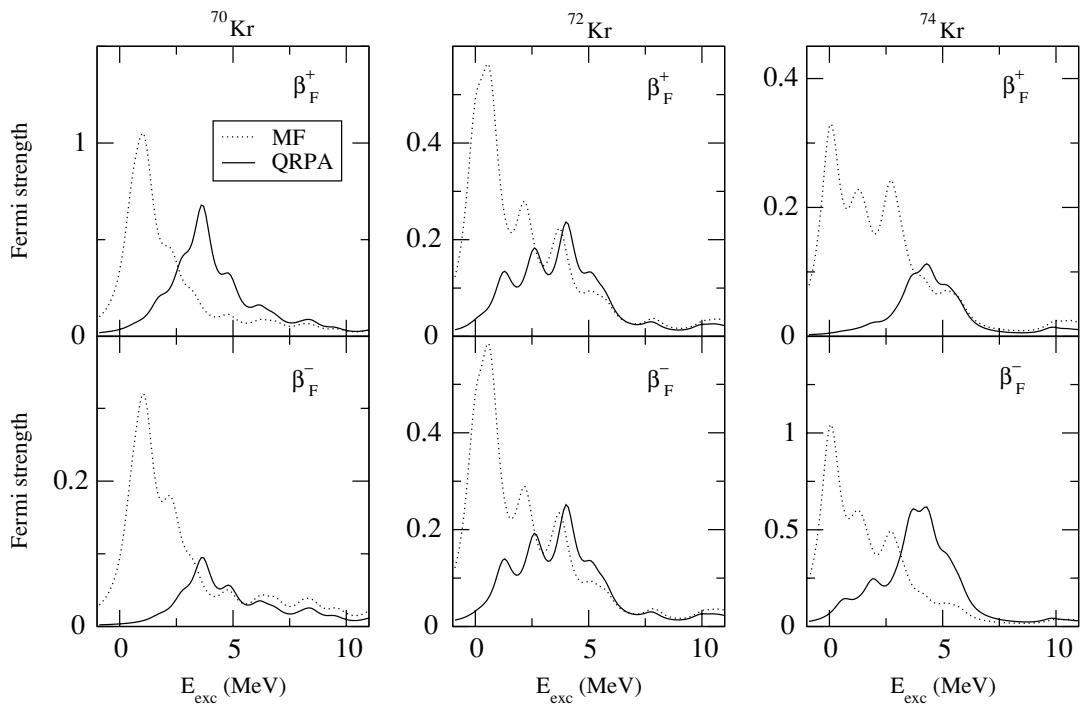

FIG. 5. Same as in Fig. 4 but including Coulomb interaction.

perpendicular to the symmetry axis $z$; that is,

$$
\left\langle J_{\perp}^{2}\right\rangle \equiv\left\langle J^{2}\right\rangle-\left\langle J_{z}\right\rangle^{2}=\frac{1}{2} \sum_{f}\left|\left\langle f\left|J_{+}\right| 0\right\rangle\right|^{2}+\left|\left\langle f\left|J_{-}\right| 0\right\rangle\right|^{2}
$$

Similarly, taking the $z$ axis in isospin space in the standard way $\left[\hat{T}_{z}=(\hat{N}-\hat{Z}) / 2\right.$, or $\left.T_{z}=(N-Z) / 2\right]$, we can measure the amount of isospin mixing by the expectation value of $T_{\perp}^{2}=$ $T_{x}^{2}+T_{y}^{2}$, or

$$
\left\langle T_{\perp}^{2}\right\rangle=\frac{1}{2} \sum_{f}\left(\left|\left\langle f\left|T_{+}\right| 0\right\rangle\right|^{2}+\left|\left\langle f\left|T_{-}\right| 0\right\rangle\right|^{2}\right)
$$

Using the definition of Fermi transition amplitudes in Eqs. (2.3) and (2.4), one immediately gets the identity

$$
\left\langle T_{\perp}^{2}\right\rangle=\frac{1}{2}\left(S_{F^{+}}+S_{F^{-}}\right)
$$

with

$$
S_{F^{ \pm}}=\sum_{f}\left|F_{f}^{ \pm}\right|^{2}=\sum_{f} B_{f}^{F^{ \pm}}
$$

In Tables I and II we compare $\left\langle T_{\perp}^{2}\right\rangle$ values to $\left\langle J_{\perp}^{2}\right\rangle$ values. The $\left\langle J_{\perp}^{2}\right\rangle$ values are calculated [2] using the expression

$$
\begin{aligned}
\left\langle J_{\perp}^{2}\right\rangle= & \sum_{\tau=p, n} \sum_{i \tau k \tau}\left(u_{i \tau} v_{k \tau}-u_{k \tau} v_{i \tau}\right)^{2} \\
& \times\left[\left|\left\langle i_{\tau}\left|j_{+}\right| k_{\tau}\right\rangle\right|^{2}+\frac{1}{2}\left|\left\langle i_{\tau}\left|j_{+}\right| \bar{k}_{\tau}\right\rangle\right|^{2}\right] .
\end{aligned}
$$

Table I corresponds to calculations in the no-pairing limit $(\Delta=0)$. Table II contains the results obtained taking into account BCS pairing correlations with realistic values of the pairing gaps (listed in the tables).

To see the amount of isospin mixing introduced by the Coulomb force we give $\left\langle T_{\perp}^{2}\right\rangle$ values obtained with and without the Coulomb force.

As seen in the tables, the values of $\left\langle J_{\perp}^{2}\right\rangle$ are large for the deformed isotopes. They decrease with decreasing deformation and become $0\left(\left\langle J_{\perp}^{2}\right\rangle=0\right)$ for spherical isotopes $\left({ }^{76} \mathrm{Kr},{ }^{78} \mathrm{Kr}\right)$.

Comparing the $\left\langle J_{\perp}^{2}\right\rangle$ values in Tables I and II, one can see that $\left\langle J_{\perp}^{2}\right\rangle$ decreases with pairing. In the no-pairing limit for the

\begin{tabular}{|c|c|c|c|c|c|c|c|}
\hline & \multirow[t]{2}{*}{$\beta$} & \multirow[t]{2}{*}{$\left\langle J_{\perp}^{2}\right\rangle$} & \multicolumn{2}{|c|}{$\left\langle T_{\perp}^{2}\right\rangle_{0}$} & \multicolumn{2}{|c|}{$\left\langle T_{\perp}^{2}\right\rangle$} & \multirow[t]{2}{*}{ IKEDA } \\
\hline & & & Coulomb & No Coulomb & Coulomb & No Coulomb & \\
\hline${ }^{72} \mathrm{Kr}$ & -0.29 & 63.1 & 0.05 & 0.00 & 0.05 & 0.00 & 0.00 \\
\hline \multirow[t]{2}{*}{${ }^{74} \mathrm{Kr}$} & -0.15 & 21.1 & 0.05 & 0.01 & 1.05 & 1.01 & 2.00 \\
\hline & 0.39 & 89.9 & 0.06 & 0.02 & 1.06 & 1.02 & 2.00 \\
\hline
\end{tabular}
prolate shape of ${ }^{74} \mathrm{Kr}$, where the deformation value reaches the value $\beta \simeq 0.4$, one gets $\left\langle J_{\perp}^{2}\right\rangle \simeq 90$. Pairing correlations with realistic gap values reduce this large $\left\langle J_{\perp}^{2}\right\rangle$ value by one third.

TABLE I. Comparison of angular momentum and isospin mixing in the mean field approximation without pairing correlations (HF). The $\mathrm{HF}$ value of the $\beta$-deformation parameter is given in the second column. 
TABLE II. Same as Table I taking into account pairing correlations (HF+BCS) with fixed pairing gaps $\Delta_{p}=\Delta_{n}=\Delta$ given in the second column.

\begin{tabular}{|c|c|c|c|c|c|c|c|c|}
\hline & \multirow[t]{2}{*}{$\Delta(\mathrm{MeV})$} & \multirow[t]{2}{*}{$\beta$} & \multirow[t]{2}{*}{$\left\langle J_{\perp}^{2}\right\rangle$} & \multicolumn{2}{|c|}{$\left\langle T_{\perp}^{2}\right\rangle_{0}$} & \multicolumn{2}{|c|}{$\left\langle T_{\perp}^{2}\right\rangle$} & \multirow[t]{2}{*}{ IKEDA } \\
\hline & & & & Coulomb & No Coulomb & Coulomb & No Coulomb & \\
\hline${ }^{70} \mathrm{Kr}$ & 1.5 & -0.23 & 28.5 & 1.2 & 1.1 & 2.2 & 2.1 & -2.0 \\
\hline${ }^{72} \mathrm{Kr}$ & 1.5 & -0.24 & 31.8 & 2.2 & 2.2 & 2.2 & 2.2 & 0.0 \\
\hline \multirow[t]{2}{*}{${ }^{74} \mathrm{Kr}$} & 1.5 & -0.15 & 13.0 & 1.5 & 1.4 & 2.5 & 2.4 & 2.0 \\
\hline & & 0.39 & 59.9 & 1.3 & 1.3 & 2.3 & 2.3 & 2.0 \\
\hline${ }^{76} \mathrm{Kr}$ & 1.65 & 0.0 & 0.0 & 1.3 & 1.2 & 3.3 & 3.2 & 4.0 \\
\hline${ }^{78} \mathrm{Kr}$ & 1.75 & 0.0 & 0.0 & 1.0 & 0.9 & 4.0 & 3.9 & 6.0 \\
\hline
\end{tabular}

In contrast, $\left\langle T_{\perp}^{2}\right\rangle$ values increase when pairing correlations are included. The large $\left\langle J_{\perp}^{2}\right\rangle$ values for the well-deformed shapes are in contrast with the small values of $\left\langle T_{\perp}^{2}\right\rangle_{0}$, with which they are to be compared. The $\left\langle T_{\perp}^{2}\right\rangle_{0}$ values, given in the tables along with $\left\langle T_{\perp}^{2}\right\rangle$ values, are defined as

$$
\left\langle T_{\perp}^{2}\right\rangle_{0}=\left\langle T_{\perp}^{2}\right\rangle-\left|\frac{N-Z}{2}\right|
$$

To clarify the meaning of this magnitude, we recall that in the limit in which the ground state is an isospin eigenstate, with $T=\left|T_{z}\right|=|(N-Z) / 2|$, we have that

$$
\left\langle T_{\perp}^{2}\right\rangle=\left[T(T+1)-T_{z}^{2}\right]=\left|T_{z}\right|=\left|\frac{N-Z}{2}\right| .
$$

Therefore $\left\langle T_{\perp}^{2}\right\rangle_{0}$ gives a better measure of the isospin mixing than $\left\langle T_{\perp}^{2}\right\rangle$ in isotopes with $|N-Z| \neq 0$.

We see in Table I that without pairing or Coulomb forces $\left\langle T_{\perp}^{2}\right\rangle=\left\langle T_{\perp}^{2}\right\rangle_{0}=0$ in $N=Z$ isotopes (i.e., there is no isospin mixing in the HF approach when an isospin-conserving twobody force is used). The amount of isospin mixing introduced by the inclusion of the Coulomb force in the Hartree-Fock approximation can be seen by comparing the $\left\langle T_{\perp}^{2}\right\rangle_{0}$ values with and without the Coulomb force in Table I. For the $N \neq Z$ isotopes in the HF approach the amount of isospin mixing as measured by $\left\langle T_{\perp}^{2}\right\rangle_{0}$ is on the order of a few percent without the Coulomb force and gets increases somewhat when the Coulomb force is included. It is interesting to see that the $\left\langle T_{\perp}^{2}\right\rangle_{0}$ value tends to decrease with increasing $\left|T_{z}\right|=\mid(N-$ $Z) / 2$ | value. This agrees with spectroscopic observations and confirms the idea that the larger the value of $|N-Z|$, the better the isospin quantum number is.

As already mentioned, pairing correlations increase isospin mixing, which as seen in Table II reaches a maximum in the $N=Z^{72} \mathrm{Kr}$ isotope where $\left\langle T_{\perp}^{2}\right\rangle_{0} \simeq 2.2$.

Yet if we compare this latter value with the $\left\langle J_{\perp}^{2}\right\rangle$ value $\left(\left\langle J_{\perp}^{2}\right\rangle \sim 32\right)$ for the same nucleus, we see that one cannot really talk of an isospin rotational band. Indeed, if we write the $\mathrm{HF}+\mathrm{BCS}$ state $\phi$ of ${ }^{72} \mathrm{Kr}$ as a linear combination of isospin eigenstates $\phi_{T}$,

$$
\phi=\sum_{T} C_{T} \phi_{T}, \quad \sum_{T}\left|C_{T}\right|^{2}=1,
$$

one sees that the mixing of $T \neq 0$ components is quite insignificant. Indeed, $\left\langle T_{\perp}^{2}\right\rangle \simeq 2$ means that

$$
\left\langle T_{\perp}^{2}\right\rangle=6\left|C_{2}\right|^{2}+20\left|C_{4}\right|^{2}+\cdots \simeq 2
$$

where one sees that $\sum_{T>0}\left|C_{T}\right|^{2} \lesssim 0.3$ and $\left|C_{2(n+1)}\right|^{2}<\left|C_{2 n}\right|^{2}$.

A corresponding expansion of $\phi$ in orthonormalized angular momentum eigenfunctions

$$
\phi=\sum_{J} A_{J} \varphi_{J}, \quad \sum_{J}\left|A_{J}\right|^{2}=1,
$$

gives

$\left\langle J_{\perp}^{2}\right\rangle=6\left|A_{2}\right|^{2}+20\left|A_{4}\right|^{2}+\cdots \simeq\left\{\begin{array}{l}32 \text { for } \Delta=1.5 \mathrm{MeV}, \\ 63 \text { for } \Delta=0,\end{array}\right.$

which provides no constraint on the amount of $J=2,4$ angular momentum components. This exercise shows that although angular momentum projection can be used to build up a rotational band, isospin projection can hardly be used to construct an isospin rotational band out of $\phi$.

Let us now consider what happens when we calculate the expectation values of $T_{\perp}^{2}$ in the $p n$-QRPA-correlated ground state. The results obtained when we take into account QRPA correlations induced by Fermi residual interactions of the form $\chi_{F}\left(\vec{\tau}_{1} \cdot \vec{\tau}_{2}\right)$ can be seen in Tables III and IV. In these tables we give the values obtained for the total strengths $S_{F^{+}}$and $S_{F^{-}}$together with $\left\langle T_{\perp}^{2}\right\rangle_{0}$ with and without Coulomb interaction.

In the QRPA approximation the total strengths for $\beta^{+}$and $\beta^{-}$Fermi transitions are given by Eq. (3.4), where the sum over $f$ runs over all the $p n$-QRPA solutions and the $\beta^{ \pm}$amplitudes $F_{f}^{ \pm}$are given by Eq. (2.7).

The QRPA solutions [17] satisfy the orthonormalization conditions

$$
\begin{gathered}
2 \sum_{f}\left(X_{i^{\prime} n i p}^{f} X_{j^{\prime} n j p}^{f *}-Y_{i^{\prime} n i p}^{f} Y_{j^{\prime} n j p}^{f *}\right)=\delta_{i j} \delta_{i^{\prime} j^{\prime}}, \\
\sum_{f}\left(X_{i^{\prime} n i p}^{f} Y_{j^{\prime} n j p}^{f *}-Y_{i^{\prime} n i p}^{f} X_{j^{\prime} n j p}^{f *}\right)=0 .
\end{gathered}
$$


TABLE III. Results of QRPA calculations of total $\beta^{+}$and $\beta^{-}$Fermi strengths of Kr isotopes, with and without Coulomb interaction, in the small pairing limit $(\Delta=0.1 \mathrm{MeV})$. Also listed are the values of $\left\langle T_{\perp}^{2}\right\rangle_{0}$. The values of the deformation parameter $\beta$ are as in Tables I and II. The strengths of the particle-hole and particle-particle residual interactions are $\chi_{p h}^{F}=0.5 \mathrm{MeV}$ and $\kappa_{p p}^{F}=0.03 \mathrm{MeV}$, respectively (see Ref. [17]).

\begin{tabular}{|c|c|c|c|c|c|c|}
\hline \multirow[t]{2}{*}{ Isotope } & \multicolumn{2}{|c|}{$S_{F^{-}}$} & \multicolumn{2}{|c|}{$S_{F^{+}}$} & \multicolumn{2}{|c|}{$\left\langle T_{\perp}^{2}\right\rangle_{0}$} \\
\hline & Coulomb & No Coulomb & Coulomb & No Coulomb & Coulomb & No Coulomb \\
\hline${ }^{72} \mathrm{Kr}$ & 0.07 & 0.02 & 0.07 & 0.02 & 0.07 & 0.02 \\
\hline \multirow[t]{2}{*}{${ }^{74} \mathrm{Kr}$} & 2.03 & 2.00 & 0.03 & 0.00 & 0.03 & 0.00 \\
\hline & 2.05 & 2.00 & 0.05 & 0.00 & 0.05 & 0.00 \\
\hline
\end{tabular}

Using these orthonormalization conditions, one can show that

$$
\begin{aligned}
S_{F^{+}}=Z-2 \sum_{i i^{\prime}}\left\langle i^{\prime} \mid i\right\rangle^{*}\{ & \left\langle i^{\prime} \mid i\right\rangle v_{i^{\prime} n}^{2} v_{i p}^{2}-\sum_{f}\left(Y_{i^{\prime} n i p}^{f}\right) \\
& \left.\times\left[v_{i^{\prime} n} u_{i p} F_{f}^{+}+u_{i^{\prime} n} v_{i p} F_{f}^{-}\right]\right\}, \\
S_{F^{-}}=N-2 \sum_{i i^{\prime}}\left\langle i^{\prime} \mid i\right\rangle^{*}\left\{\left\langle i^{\prime} \mid i\right\rangle v_{i^{\prime} n}^{2} v_{i p}^{2}-\sum_{f}\left(Y_{i^{\prime} n i p}^{f}\right)\right. & \\
& \left.\times\left[v_{i^{\prime} n} u_{i p} F_{f}^{+}+u_{i^{\prime} n} v_{i p} F_{f}^{-}\right]\right\},
\end{aligned}
$$

with $F_{f}^{ \pm}$as given in Eq. (2.7).

These equations explicitly show that, as pointed out in Ref. [21], the total $\beta^{+}\left(\beta^{-}\right)$strength splits into a one-body term that counts the total number of protons (neutrons) and a two-body term that depends on the two-body correlations included in the descriptions of the nuclear ground state. The two-body term is identical in $F^{+}$and $F^{-}$summed strengths, and as a result one gets the Ikeda sum rule

$$
S_{F^{-}}-S_{F^{+}}=N-Z,
$$

which is always satisfied in our calculations.
The last term in Eqs. (3.14) and (3.15) is the QRPA correlation term

$$
C_{\mathrm{QRPA}}=2 \sum_{f} \sum_{i p i^{\prime} n}\left(Y_{i p i^{\prime} n}^{f}\right)\left\langle i^{\prime} \mid i\right\rangle^{*}\left[v_{i^{\prime} n} u_{i p} F_{f}^{+}+u_{i^{\prime} n} v_{i p} F_{f}^{-}\right] .
$$

This term is zero when there are no QRPA correlations. If the strength of the residual interaction is zero $\left(\chi_{F}=0\right)$, the $Y$ amplitudes are zero.

The second term in Eqs. (3.14) and (3.15) contains the pure pairing correlation and it reaches its minimum value in the limit of no pairing and no Coulomb force. In the HF limit one has that

$$
\left(-2 \sum_{i i^{\prime}}\left|\left\langle i^{\prime} \mid i\right\rangle\right|^{2} v_{i^{\prime} n}^{2} v_{i p}^{2}\right)_{\mathrm{HF}}=-2 \sum_{i<\lambda_{p}, i^{\prime}<\lambda_{n}}\left|\left\langle i^{\prime} \mid i\right\rangle\right|^{2} .
$$

If, in addition, there is no Coulomb force

$$
\left\langle i^{\prime} \mid i\right\rangle=\delta_{i^{\prime} i} \text { for } N=Z,
$$

and we have that

\begin{tabular}{|c|c|c|c|c|c|c|}
\hline \multirow[t]{2}{*}{ Isotope } & \multicolumn{2}{|c|}{$S_{F^{-}}$} & \multicolumn{2}{|c|}{$S_{F^{+}}$} & \multicolumn{2}{|c|}{$\left\langle T_{\perp}^{2}\right\rangle_{0}$} \\
\hline & Coulomb & No Coulomb & Coulomb & No Coulomb & Coulomb & No Coulomb \\
\hline${ }^{70} \mathrm{Kr}$ & 0.43 & 0.41 & 2.43 & 2.41 & 0.43 & 0.41 \\
\hline${ }^{72} \mathrm{Kr}$ & 1.15 & 1.11 & 1.15 & 1.11 & 1.15 & 1.11 \\
\hline \multirow[t]{2}{*}{${ }^{74} \mathrm{Kr}$} & 2.50 & 2.44 & 0.50 & 0.44 & 0.50 & 0.44 \\
\hline & 2.46 & 2.48 & 0.46 & 0.48 & 0.46 & 0.48 \\
\hline${ }^{76} \mathrm{Kr}$ & 4.30 & 4.23 & 0.30 & 0.23 & 0.30 & 0.23 \\
\hline${ }^{78} \mathrm{Kr}$ & 6.17 & 6.12 & 0.17 & 0.12 & 0.17 & 0.12 \\
\hline
\end{tabular}

$$
\begin{array}{r}
\left(-2 \sum_{i i^{\prime}}\left|\left\langle i^{\prime} \mid i\right\rangle\right|^{2} v_{i^{\prime} n}^{2} v_{i p}^{2}\right)_{\mathrm{HF}}=-2 \sum_{i<\lambda_{p}, i^{\prime}<\lambda_{n}} \delta_{i^{\prime} i}=-Z \\
\text { for } N=Z .
\end{array}
$$

We therefore find that, in the limit of no pairing and no Coulomb force,

$$
\left\langle T_{\perp}^{2}\right\rangle=\left\langle T_{\perp}^{2}\right\rangle_{\mathrm{HF}}=0 \quad \text { for } N=Z,
$$

TABLE IV. Same as Table III but for standard values of the pairing gap ( $\Delta=\Delta_{n}=\Delta_{p} \sim 1.5 \mathrm{MeV}$, as listed in Table II). 
as we found numerically in Table I. In the general case we can write

$$
\left\langle T_{\perp}^{2}\right\rangle=\frac{1}{2}\left(S_{F^{+}}+S_{F^{-}}\right)=\left|\frac{N-Z}{2}\right|+C_{\mathrm{BCS}}+C_{\mathrm{QRPA}},
$$

with $C_{\mathrm{QRPA}}$ the QRPA correlation term defined in Eq. (3.17) and $C_{\mathrm{BCS}}$ the BCS correlation term defined as

$$
C_{\mathrm{BCS}}=-2 \sum_{i^{\prime} n i p}\left|\left\langle i^{\prime} \mid i\right\rangle\right|^{2} v_{i^{\prime} n}^{2} v_{i p}^{2}+\min (Z, N) .
$$

Owing to Eq. (3.22) we see that the value of $\left\langle T_{\perp}^{2}\right\rangle_{0}$, as defined in Eq. (3.6), is purely due to the correlation terms in Eqs. (3.17)-(3.23),

$$
\left\langle T_{\perp}^{2}\right\rangle_{0}=\left\langle T_{\perp}^{2}\right\rangle-\left|\frac{N-Z}{2}\right|=C_{\mathrm{BCS}}+C_{\mathrm{QRPA}},
$$

and that for $N=Z$ and no Coulomb force

$$
\left\langle T_{\perp}^{2}\right\rangle=\left\langle T_{\perp}^{2}\right\rangle_{0}=\left\langle T_{\perp}^{2}\right\rangle_{\mathrm{HF}} .
$$

The results in Tables I and II show that the BCS correlations always increase the $\left\langle T_{\perp}^{2}\right\rangle$ value from its limiting Hartree-Fock value. However, if we compare the results of Tables I and II to the results of Tables III and IV, which include both correlation terms [see Eq. (3.24)], we see that the increase of $\left\langle T_{\perp}^{2}\right\rangle_{0}$ due to the BCS correlations is largely reduced by the QRPA correlations, which tend to restore isospin invariance.

\section{SUMMARY AND CONCLUDING REMARKS}

We have studied isospin mixing properties in several $\mathrm{Kr}$ isotopes around $N=Z$ and have analyzed their Fermi transitions at various levels of approximation.

We have first considered self-consistent deformed Skyrme HF mean fields with and without Coulomb and pairing interactions. Then we took into account isospin-dependent residual interactions and considered QRPA-correlated ground states. We have studied the effect on the Fermi strength distributions of isospin-breaking interactions (Coulomb force and pairing), as well as the effect of QRPA correlations including Fermi-type residual interactions, whose particlehole strengths are consistently fixed with the Skyrme force.

Taking as a reference the case of a self-consistent mean field calculation, we have seen that in the absence of Coulomb interactions (and in the limit of small pairing correlations) the isospin-forbidden transitions $\left(\beta^{-}\right.$in $N \leqslant Z$ and $\beta^{+}$in $N \geqslant Z$ ) are negligible. When the isospin-breaking Coulomb interaction is switched on, there is an increase of isospinforbidden Fermi transitions. Although this increase is small, it signifies isospin breaking. Pairing correlations increase isospin-forbidden Fermi transitions by orders of magnitude, a fact related to the isospin-breaking nature of the quasiparticle mean field, which increases with increasing pairing gaps. However, the isospin-breaking effects and forbidden Fermi transitions are reduced when RPA correlations are taken into account.

In analogy with the quantity $\left\langle J_{\perp}^{2}\right\rangle$, which measures the amount of angular momentum mixing, we have introduced the quantity $\left\langle T_{\perp}^{2}\right\rangle_{0}$ as a measure of the isospin mixing in the ground state.

In the extreme case of the mean field approach without Coulomb interaction and without pairing correlations, we have $\left\langle T_{\perp}^{2}\right\rangle_{0}=0$ for $N=Z$. There is no isospin mixing in the HF approach when isospin-conserving two-body forces are used. The amount of isospin mixing introduced by the Coulomb force is small and the maximum mixing occurs when $N=Z$. In contrast to $\left\langle J_{\perp}^{2}\right\rangle$, which decreases with increasing pairing, the amount of isospin mixing $\left\langle T_{\perp}^{2}\right\rangle_{0}$ increases with pairing correlations and it is also maximum for $N=Z$. The lowest isospin mixing, as measured by $\left\langle T_{\perp}^{2}\right\rangle_{0}$, occurs in the HF approximation. Pairing correlations increase the mixing and QRPA correlations reduce it.

One may wonder whether, in deformed nuclei, the calculated Fermi strengths may contain spurious contributions from higher angular momentum components in the initial and final wave functions. As mentioned before, the Fermi strengths are calculated in the laboratory frame in the factorization approximation of Bohr and Mottelson [5]. Using angular momentum projection techniques [2], we find that an upper bound to such contributions is proportional to $1 /\left\langle J_{\perp}^{2}\right\rangle^{2}$, where the values of $\left\langle J_{\perp}^{2}\right\rangle$ can be found in Tables I and II. Thus, exact angular momentum projection in the deformed cases would lead in all cases to less than a $1 \%$ effect in the Fermi strengths.

We have also shown that the total $\beta^{ \pm}$Fermi strengths can be separated into a one-body term, counting basically the number of nucleons of a given type, and a two-body term that depends on the two-body correlations (BCS and RPA). The two-body term is identical in both $\beta^{+}$and $\beta^{-}$summed strengths and the Ikeda sum rule is fulfilled as a result of their cancellation. The isospin mixing $\left\langle T_{\perp}^{2}\right\rangle_{0}$ is purely due to the net effect of the correlation terms.

\section{ACKNOWLEDGMENTS}

This work was supported by Ministerio de Educación y Ciencia (Spain) under Contract No. BFM2002-03562. One of us (R.A.-R.) thanks Ministerio de Educación y Ciencia (Spain) for financial support.
[1] A. de Shalit and H. Feshbach, Theoretical Nuclear Physics (Wiley, New York, 1974); P. Ring and P. Schuck, The Nuclear Many-Body Problem (Springer-Verlag, New York, 1980); I. Talmi, Simple Models of Complex Nuclei (Harwood Academic, Chur, Switzerland, 1993).
[2] E. Moya de Guerra, Phys. Rep. 138, 293 (1986).

[3] H. Flocard, P. Quentin, and D. Vautherin, Phys. Lett. B46, 304 (1973); A. Zarringhalam and J. W. Negele, Nucl. Phys. A288, 417 (1977); E. Moya de Guerra and S. Kowalski, Phys. Rev. C 20, 357 (1979). 
[4] F. Villars, in Many-Body Description of Nuclear Structure and Reactions, edited by C. Bloch (Academic Press, New York, 1966), p. 1.

[5] A. Bohr and B. Mottelson, Nuclear Structure (Benjamin, New York, 1975).

[6] M. J. Musolf and T. W. Donnelly, Nucl. Phys. A546, 509 (1992); T. W. Donnelly (private communication).

[7] I. S. Towner and J. C. Hardy, Phys. Rev. C 66, 035501 (2002).

[8] A. A. Raduta and E. Moya de Guerra, Ann. Phys. (NY) 284, 134 (2000).

[9] W. Satula and R. A. Wyss, Acta Phys. Pol. B 32, 2441 (2001); S. Glowacz, W. Satula, and R. A. Wyss, Eur. Phys. J. A 19, 33 (2004).

[10] A. P. Zuker, S. M. Lenzi, G. Martínez-Pinedo, and A. Poves, Phys. Rev. Lett. 89, 142502 (2002).

[11] D. Vautherin and D. M. Brink, Phys. Rev. C 5, 626 (1972);

D. Vautherin, ibid. 7, 296 (1973); M. Vallieres and D. W. L. Sprung, Can. J. Phys. 56, 1190 (1978).

[12] N. Auerbach, Phys. Rep. 98, 273 (1983); N. Auerbach and O. K. Vorov, Phys. Lett. B414, 1 (1997).

[13] I. Hamamoto and H. Sagawa, Phys. Rev. C 48, 960(R) (1993); J. Dabaczewski and I. Hamamoto, Phys. Lett. B345, 181 (1995);
H. Sagawa, N. Van Giai, and T. Suzuki, Phys. Rev. C 53, 2163 (1996); G. Colo, M. A. Nagarajan, P. Van Isacker, and A. Vitturi, ibid. 52, 1175(R) (1995).

[14] G. F. Bertsch and S. F. Tsai, Phys. Rep. 18, 126 (1975).

[15] A. A. Raduta, L. Pacearescu, V. Baran, P. Sarriguren, and E. Moya de Guerra, Nucl. Phys. A675, 503 (2000).

[16] A. A. Raduta, P. Sarriguren, A. Faessler, and E. Moya de Guerra, Ann. Phys. (NY) 294, 182 (2001).

[17] P. Sarriguren, E. Moya de Guerra, A. Escuderos, and A. C. Carrizo, Nucl. Phys. A635, 55 (1998).

[18] P. Sarriguren, E. Moya de Guerra, and A. Escuderos, Nucl. Phys. A658, 13 (1999); A691, 631 (2001); Phys. Rev. C 64, 064306 (2001).

[19] N. Van Giai and H. Sagawa, Phys. Lett. B106, 379 (1981).

[20] E. Caurier, A. Poves, and A. P. Zuker, Phys. Rev. Lett. 74, 1517 (1995); E. Caurier, G. Martinez-Pinedo, A. Poves, and A. P. Zuker, Phys. Rev. C 52, 1736(R) (1995); P. B. Radha, D. J. Dean, S. E. Koonin, K. Langanke, and P. Vogel, ibid. 56, 3079 (1997); A. Poves (private communication).

[21] E. Moya de Guerra, A. A. Raduta, L. Zamick, and P. Sarriguren, Nucl. Phys. A727, 3 (2003). 